\title{
La violence des orages dans ses relations avec le débit des égouts urbains
}

\section{The violence of storms in relation to the discharge of urban drains}

\author{
PAR P. KOCH \\ Drrecteur teghroue des Eaux et de l'Assainissement a la Préfecture de la Serne \\ Ingéneur en Chef des Pon'ts et Grausstes \\ Membre du Consert. Supérnteur d'Hygüine Publigue de France
}

English synopsis p. 636

Les égouts pluviaux ou unitaires peuvent être considérés comme des torrents à échelle réduite dont les plus fortes crues sont provoquées en règle générale par les précipitations orageuses. La durée « critique » de ces précipitations est en rapport, dans chaque cas d'espèce, avec le «temps de concentration » du ruissellement sur le bassin desservi par l'ouvrage que l'on considère (1). Pratiquement on est conduit à envisager une gamme de précipitations dont la persistance s'échelonne entre une dizaine à une vingtaine de minutes et plus d'une heure (dans le cas des très grandes agglomérations : région parisienne par exemple).

Les données pluviométriques que l'on considère habituellement en hydrologie et qui ont trait à la hauteur d'eau recueillie annuellement, à sa répartition suivant les saisons et les mois, aux nombres de jours de pluie correspondants, etc., ne présentent guère d'intérêt pour les techniciens chargés d'établir les projets d'assainissement urbain.

Les éléments utiles à ces techniciens concernent la répartition des averses dans le cadre de

(1) Le temps de concentration se définit comme la durée mise par la goutte d'eau la plus éloignée (en temps d'écoulement) pour parvenir au débouché du bassin en cause. l'heure ou tout au plus des 24 heures. Ce dernier laps de temps est celui dont les indications sont domnées directement par les pluviomètres ordinaires, que l'on relève une fois par jour. On doit en compléter l'effet par les notes d'observateurs qui pointent les heures des débuts et des fins d'averses aux points particuliers où ils siègent d'une manière permanente.

Un certain nombre d'observatoires sont dotés, quant à eux, de pluviomètres enregistreurs dont le tambour, au diamètre généralement voisin de dix centimètres, fait un tour complet en 24 heures. Sur les graphiques obtenus, un orage violent se traduit par une remontée importante et rapide du trait sur quelques centimètres, entre deux abscisses distantes de quelques millimètres. Assez précis quant à la mesure des hauteurs d'eau recueillies, de tels relevés le sont moins pour l'appréciation de la durée des averses très courtes ou pour l'analyse des avaJaisons plus prolongées suivant leurs différentes phases.

Les statistiques publiées par les observatoires se bornent d'ailleurs le plus souvent à relater la durée des orages enregistrés et la hauteur d'eau fournie au total par chacun d'eux (sinon au cours de la journée où ils se sont produits).

Ces indications sont déjà précieuses pour le 
technicien sanitaire, lor'squ'elles s'étendent sur une période suffisante. Leur report sur un plan de coordonnées permet d'étudier le groupement des points représentatifs; on peut ensuite s'en inspirer soit pour dégager une « courbe enveloppe » des plus forts débits observés, soit pour tracer à vue des courbes caractéristiques des orages « notables », « remarquables» ou « très rares » (1); ces vocables ne sauraient d'ailleurs répondre à une notion vraiment précise de la «fréquence »du fait que celle-ci, définie d'après le nombre des précipitations dont les durées se sont échelonnées entre $\mathrm{T}_{0}$ et $\mathrm{T}_{0}+\Delta \mathrm{T}$, apparait à la fois comme fonction de $T_{0}$ et $d u$ paramètre arbitraire $\Delta \mathrm{T}$.

Il n'en reste pas moins que, selon le degré de protection que l'on attend des égouts à construire, on peut s'imposer l'ajustement de leur capacité d'évacuation à l'une ou l'autre de ces courbes caractéristiques.

C'est ainsi qu'un grand nombre de réseaux ont été calculés en Amérique sur la base de la formule de TALBO'T qui, transposée en unités métriques ( $\mathrm{H}$ en millimètres, $\mathrm{T}$ restant décompté en minutes), se traduit par l'expression:

$$
i_{m}=\frac{\mathrm{H}}{\mathrm{T}}=\frac{44}{\mathrm{~T}+15}
$$

considérée comme représentant une courbe d'orages relativement peu fréquents.

Pour la région parisienne en particulier, nous avons été amené vers 1930 à dégager la formule

$i_{m}=\frac{57}{\mathrm{~T}+15}$, dérivée de la précédente, mais

qui donne avec $\mathrm{T}=60$ minutes la \& pluie BeLGRAND $\gg$ de $45 \mathrm{~mm}$ à l'heure prise en compte par ce précurseur pour le calcul des grands collecteurs de la Capitale. Dans la pratique, l'élément qui importe principalement au technicien sanitaire consiste non pas en l'intensité moyenne de la précipitation sur l'ensemble de sa durée, mais en celle que l'on observe au cours de sa phase la plus violente, sur une période suffisante eu égard au "temps de concentration »du ruissellement pour le bassin à drainer. Mais, s'agissant de pareilles déterminations, les graphiques enregistrés sur les pluviomètres habitucls apparaissent comme insuffisants, au moins pour les durées sensiblement inférieures à une demiheure, qui entrent le plus souvent en ligne de

(1) Terminologie adoptée par l'office Météorologique de Grande-Bretagne avant la guerre. compte; ils comportent en effet une échelle des abscisses trop faible pour permettre une analyse fructueuse dans des limites aussi étroites.

C'est seulement en 1926 que le Service d'Etudes et de Statistiques Climatiques de la Ville de Paris s'est avisé d'installer à l'Observatoire de Montsouris un enregistreur à marche rapide, faisant piusieurs tours en 24 heures au lieu d'un seul.

En 1947, à notre demande, M. Grisollet, actuellement Chef de ce Service, a procédé au dépouillement des observations recueillies depuis l'origine, en classant par valeurs décroissantes les intensités moyennes constatées sur 10, 15, 20,25 , 30 minutes... au cours de la phase la plus violente des orages correspondants. Ce dépouillement a permis de dégager, pour chaque durée ' $\mathrm{T}_{0}$, une série de valeurs $i_{m}$ traduisant, sur des bases rationnelles, la cadence observable en moyenne respectivement une fois par an, tous les 5 ans, tous les 10 ans, etc... Les cadences correspondant à une même fréquence, pour les différentes valeurs de $T$, définizsent une courbe $i_{m}=f(\mathrm{~T})$ caractéristique de cette fréquence.

La même analyse a été faite, pour des durées de $30,40,50,60$ minutes, d'après des enregistrements accumulés depuis 1897 sur les appareils à faible vitesse de rotation.

M. Grisollet a été ainsi amené à dresser, comme représentatif des orages observés à Montsouris, un faisceau d'hyperboles équilatères dont l'équation et les paramètres ressortent comme suit ( $\dot{i}_{m}$ étant ici exprimé en $m m$ à l'heure et $\mathrm{T}$ en minutes):

AVERSE-TYPE DÉPASSÉE OU ATTEINTE UNE FOIS EN :

\begin{tabular}{|c|c|c|c|c|}
\hline & 10 ans & 5 ans & 2 ans & 1 an \\
\hline$i_{m}=\frac{a}{b+\mathrm{T}}\left\{\begin{array}{l}a= \\
b=\end{array}\right.$ & $\begin{array}{r}2590 \\
10\end{array}$ & $\begin{array}{r}2100 \\
9\end{array}$ & $\begin{array}{r}1400 \\
7\end{array}$ & $\begin{array}{r}830 \\
5\end{array}$ \\
\hline
\end{tabular}

Il n'est pas sans intérêt d'établir un rapprochement. entre ces données, solidement appuyées sur l'expérience, et les résultats que foumirait en l'espèce l'application de la loi d'effet proportionnel établie par M. Gibrat, selon les modalités qu'a suggérées M. CAQUot en 1941. Sur la base de ces suggestions, on devrait pouvoir. dégager des constantes $a, b, c$, telles que la grandeur $z=a \log \mathrm{H}+b \log \mathrm{T}+c$ satisfasse à la loi de Gauss. Il s'en déduirait que les courbes $\mathrm{H}=f(\mathrm{~T})$ correspondant à diverses fréquences admettraient des équations de la forme $\mathrm{H}=\mathrm{T}^{\beta}$, ou $\alpha$ représenterait le seul paramètre correspondant à la fréquence.

M. Caguot a montré le parti pratique que l'on pourrait tirer de telles expressions pour établir 
des formules commodément adaptables au calcul direct du ruissellement en provenance de bassins urbanisés.

Sans doute les récents travaux de M. GrisolLET n'ont pas confirmé formellement la validité de la formule monome en question, laquelle exigerait notamment que les courbes représentant les averses de différentes fréquences découlent les unes des autres par simple dilatation ou contraction des ordonnées.

Toutefois, sur le plan théorique, on doit souligner que cette pierre d'achoppement ne saurait par elle-même mettre en échec dans son essence la loi de l'effet proportionnel, laquelle s'accommoderait d'une formule en $z$ dont le type général serait:

$$
\begin{gathered}
a^{\prime} \log \left(\mathrm{H}-\mathrm{H}_{0}\right) \log \left(\mathrm{T}-\mathrm{T}_{0}\right) \\
+b^{\prime} \log \left(\mathrm{H}-\mathrm{H}_{0}\right)+c^{\prime} \log \left(\mathrm{T}-\mathbf{T}_{0}\right)+d^{\prime}
\end{gathered}
$$

Si l'on acceptait dès lors de renoncer à la formule en $z$ simplifiée qu'a suggérée M. CAqvot et si l'on se ménageait l'introduction de paramètres supplémentaires dans une expression plus complexe, rien n'empêcherait sans doute de faire concorder la réalité et la théorie avec toute la précision désirable.

Mais, sur le plan pratique, on serait obligé de renoncer pour autant aux conséquences que M. CAQuot a tirées de la formule simplifiée, et cela sans bénéfice réellement appréciable : car da recherche d'une approximation rigoureuse ne se justifierait pas dans ce calcul des apports pluviaux, étant donné l'imprécision inhérente à l'appréciation des coefficients de ruissellement.

Aussi les Instructions Techniques du 22 février 1949 sur l'Assainissement, contresignées par les Ministres de la Reconstruction et de l'Urbanisme, de l'Intérieur et de la Santé Publique, ont-elles délibérément admis les formules préconisées par M. CaQuot et se sont-elles bornées à en réévaluer les paramètres numériques sur la base des données expérimentales qu'a précisées récemment M. Grisollet.

D'autres recherches se poursuivent sur les caractères des orages qui sont à l'origine des pointes de flot dans les égouts unitaires ou pluviaux.

Une extension des périodes d'observation devra notamment permettre de préciser Timportance qu'est susceptible de prendre, en valeur moyenne, le coefficient d'intensification des orages pour les très faibles fréquences. Actuellement on se trouve en présence des échelles

\begin{tabular}{|c|c|c|c|}
\hline & 50 ans & 10 ans & 2 ans \\
\hline la Truyère) & 1,4 & 1. & 0,7 \\
\hline Caguot (orages parisiens). & 1,57 & 1. & 0,59 \\
\hline GrisollET (orages paris.). & 1,25 & 1 & 0,60 \\
\hline
\end{tabular}
suivantes :
Il reste également à poursuivre une comparaison circonstanciée entre les statistiques d'orages afférentes respectivement à Paris et aux autres régions du pays.

Sur nos suggestions, M. Grisollet s'emploie présentement à obtenir du Directeur de la Station de Bioclimatologie Agricole de Montpellier qu'il déponille dans ce sens, au moins pour les orages de durée égale ou supérieure à $30 \mathrm{mi}-$ nutes, les enregistrements à faible vitesse de rotation dont il dispose (1).

Ainsi notre connaissance du climat, sous l'aspect particulier des orages violents qui intéressent au premier chef les techniciens sanitaires, s'étend et s'affermit progressivement. On peut noter avec une certaine satisfaction que la théorie du ruissellement, née en France avec Belgrand, et qui s'était développée depuis lors surtout en Allemagne et anx Etats-Unis (2), vient de retrouver dans notre pays les éléments d'une évolution dont le parallele ne se manifeste pas ailleurs, à notre connaissance; après que nous avons eu l'occasion d'éveiller l'intérêt du problème (2), les Services Météorologiques de Paris, en les personnes de leurs chefs successifs, M. Besson (3) et M. Grisollet (4) ont poursuivi les recollements des observations pluviométriques et tenté d'en dégager la physionomie des phénomènes, tandis que $M$. CAQuot, s'appuyant sur la loi de M. Gibrat, imaginait un mode de calcul forfaitaire $d u$ ruissellement (5) qui, moyennant certaines adaptations pour tenir compte des relevés pluviométriques ultérieurs, fournit une méthode commodément applicable aux zones urbanisées (6).

(1) On peut indiquer dès à présent que l'orage type parait $y$ dépasser la durée de l'ordre de 20 minutes, qui est le plus couramment observée dans la région parisienne par exemple, avec des intensités moyennes au plus égales ou légèrement inférieures pour de mèmes intervalles de temps.

(2) Voir Annales des Ponts et Chaussées, avril 1930, et Technique Sanitaire et Municipale, juillet et août 1933 .

(3) Voir Annales des Services T'echniques d'IIygiène de la Ville de Paris, t. XIII, 1932.

(4) Voir Compte Rendu à l'Académie des Sciences, 1947, $\mathrm{n}^{\circ} 24$, et $\mathrm{n}^{\circ}$ de juillet-septembre 1948 de la Météorologie.

(5) Voì Compte Rendu à l'Acađémie des Sciences, séance du 20 octobre 1941.

(6) Voir Instructions techniques relatives à l'assainissement des agglomérations en date du 22-2-49, contresignées par les Ministres de la Reconstruction et de l'Urbanisme, de l'Intérieur et de la Santé Publique. 


\section{DIS CUSSION}

\section{Le Président remercie M. Koch.}

M. CAOUors souligne qu'aux points d'accumulation (points bas) les canalisations doivent être calculées avec précision et en admettant une probabilité minime. Il a constaté que les dimensions des canalisations étaient le plus souvent surabondantes et n'étaient jamais en rapport avec les débits probables: la formule simple qu'il a été ainsi amené à trouver dès 1908, pour la ville de Troyes, est moins précise que celles qui ont été établies par la suite, mais elle a permis de calculer des ouvrages qui, moyennant quelques retouches aux points singuliers, se sont révélés capables d'écouler les orages sans inondations.

En second lieu, la capacité des ouvrages à ces points varie suivant la vitesse de concentration, et son calcul implique la connaissance de la variation de l'intensité de l'orage dans l'espace et dans le temps selon la direction et la vitesse du vent. Cette « chronologie » de l'orage nécessite un réseau de pluviomètres-enregistreurs répartis rationnellement sur le bassin moyen d'une ville et permettrait de préciser encore les formules proposées par M. Koch.

M. CAQvor insiste sur l'importance des travaux de construction d'égouts qui sont les plus rentables pour le rombre de vies humaines qu'ils sauvent par la salubrité.

M. Косн précise que, dans sa communication d'aujourd'hui, il n'a pas abordé le problème du ruissellement évoqué par $M$. CAQUor, mais que ce problème représente le champ d'application des recherches relatives aux précipitations dans le cadre urbain. A titre d'exemple, pour vérifier s'il était légitime de tenir compte de l'entière capacité des ouvrages, M. Koch a demandé au Service Météorologicque d'étudier de très près si les pointes de précipitations se situaient bien au début de l'orage; or il semble en ètre ainsi d'une manière générale, ce qui confirme les vues dé M. CaQvot sur la portée de l'effet « capacité $\gg$, dont la méthode dite « rationnelle » des Américains présente je défaut de ne pas tenir compte, sinon a titre de coefficient de sécurité implicite.

En ce qui concerne les formules de précipitations, M. Kock rappelle que les Américains en étaient arrivés à utiliser de préférence la formule de Talbot, qui semble correspondre sensiblement à la fréquence décennale. Tou- tefois M. Kock considère qu'il convient d'attacher une importance essentielle aux définitions nuancées de frêquence qu'a préconisées notamment M. Caquot.

La fréquence décemnale paraît bien adaptée au calcul de l'ensemble d'un réseau; mais il ne faut pas hésiter à admettre des fréquences plus élevées, cinquantenaires par exemple, pour le calcul d"ouvrages singuliers tels que les déversoirs d'orage, qui ont pour but de décharger les points critiques aux ruptures de pentes et dont la longueur sénéralement limitée n'intervient pas sensiblement dans le coût total du réseau.

M. Koch indique, d'autre part, qu'un réseau de pluviomètres enregistreurs de types divers, les uns très précis pour des études poussées, les autres plus grossiers destinés à donner une indication de la répartition de la pluie d'orage dans le temps et dans l'espace, est en cours d'installation dans la région parisienne. La question de l'influence des vents sur l'accumulation à certains points bas, par exemple au pied de la butte de Vincennes, côté Charenton, a été également posée au Service Météorologique qui poursuit l'étude de ce phénomène.

Il apparaît d'ailleurs comme très désirable que des recherches analogues soient poursuivies sous d'autres «climats $\gg$; dès à présent les relevés opérés par le Service Météorologique de Montpellier montrent que les intensités y seraient plutôt plus faibles, mais que par contre les averses y dépassent assez couramment les durées de l'ordre de 20 minutes caractéristiques dans la région parisienne.

M. Hupner demande à M. Koch de bien vouloir préciser les unités employées dans la formule $i_{m}=\frac{a}{b+\mathrm{T}}$; cette précision a été apportée au texte imprimé.

M. FERRY signale un artifice d'enregistrement utilisé sur. certains pluviographes hebdomadaires suisses; ces appareils sont munis de cylindres de grande hauteur qui se déplacent d'un mouvement uniforme le long de leur axe de telle sorte que les courbes correspondant aux enregistrements journaliers ne se recouvrent pas les unes les autres.

M. le Président remercie les commentateurs de la communication de M. KocH, et particulièrement M. CAQUor.

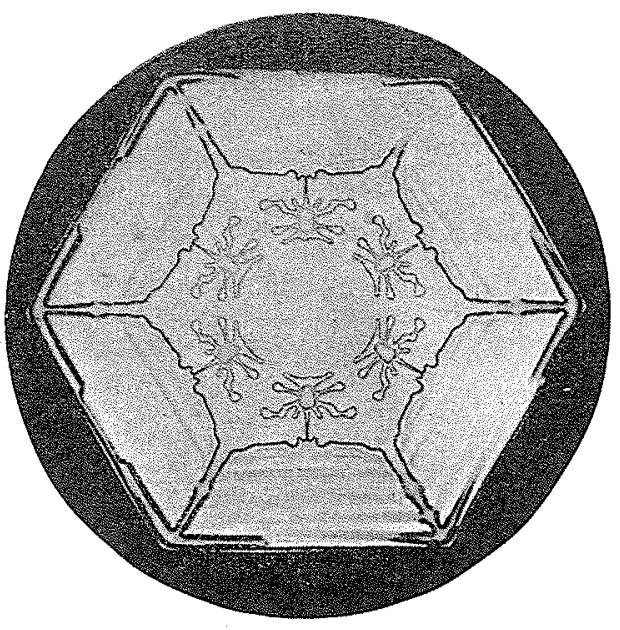

